\title{
Postcardiac Injury Syndrome After Coronary Angioplasty and Stenting
}

\author{
Christopher Hearne, MD, and Samuel N. Forjuoh, MB, ChB, DrPH
}

Pleuropericarditis has been described in many patients after a variety of cardiac insults, including infarction and surgery or trauma to the heart. The syndrome of fever, pleural effusion, chest pain, and an elevated sedimentation rate after cardiac injury is referred to as the postcardiac injury syndrome (PCIS). ${ }^{1}$ PCIS actually includes two distinct entities, the postmyocardial infarction syndrome (PMIS), which occurs after myocardial infarction, and the postcardiotomy syndrome (PCS), which occurs after cardiac surgery or trauma. We report a rare case of PCIS that occurred after an uncomplicated coronary angioplasty and stenting.

\section{Case Report}

A 79-year-old woman with multivessel coronary artery disease underwent cardiac catheterization for evaluation of accelerated angina pectoris. Her medical history included hypertension, hyperlipidemia, and peripheral vascular disease. She subsequently underwent angioplasty and stenting of the first obtuse marginal and circumflex arteries. The next day, because of recurrent angina, she underwent stenting of the distal obtuse marginal artery. When she was released from the hospital on the third day, there was no evidence of myocardial infarction.

Three weeks later, she had a low-grade fever, night sweats, myalgias, and a large left pleural effusion. The pleural effusion was an exudate. Culture and cytologic studies were negative. An echocardiogram documented a small pericardial effusion, and there were no wall motion abnormalities. An electrocardiogram showed no change from baseline and, specifically, showed no evidence of myocardial infarction. Her sedimentation rate was

Submitted, revised, 19 September 2002.

From the Departments of Internal Medicine $(\mathrm{CH})$ and Family \& Community Medicine (SNF), Scott \& White Memorial Hospital, and Scott, Sherwood and Brindley Foundation, Texas A\&M University System Health Science Center, College of Medicine, Temple, Tex. Address reprint requests to Christopher Hearne, MD, Scott \& White Georgetown Clinic, 4945 Williams Dr, Georgetown, TX 78628 . elevated to $114 \mathrm{~mm} / \mathrm{h}$ (normal less than $20 \mathrm{~mm} / \mathrm{h}$ ). She was given prednisone and improved rapidly, with resolution of the pleural effusion and normalization of the sedimentation rate. The prednisone was tapered initially over a 1 -month period, but her symptoms recurred, and she required a prolonged course of low-dose prednisone for control. Nine months later she was no longer taking steroids, and her PCIS was in remission.

\section{Discussion}

PCIS was first described in the 1950s in patients undergoing mitral commissurotomy and other cardiac surgeries. ${ }^{2}$ Dressler's syndrome, or postmyocardial infarction syndrome, pertained to patients who developed PCIS after myocardial infarction. ${ }^{3}$ Since that time, PCIS has been described as occurring after pulmonary embolism, ${ }^{4}$ pacemaker implantation, ${ }^{5}$ chest trauma, ${ }^{6}$ and a variety of cardiac insults. As was in our case, administration of corticosteroids has resulted in prompt resolution of the signs and symptoms. In fact, resolution of the signs and symptoms is so dramatic as to be diagnostic of PCIS. $^{4}$

The cause of PCIS is still unclear, although there is some evidence to support an immunologic or viral origin. ${ }^{1}$ Using a retrospective review of 35 patients, Stelzner et $\mathrm{al}^{7}$ described the clinical course of the syndrome. The onset of the syndrome was about 20 days after the injury. An elevated sedimentation rate and pleurisy were found in $96 \%$ and $91 \%$ of the patients, respectively, whereas 2 in 3 patients had a fever. Other notable signs and symptoms mentioned in the literature include hemoptysis, arthralgias, arthritis, and unexplained anemia. ${ }^{4}$ In fact, all three cases of patients in their 60 s, reported by Sklaroff, ${ }^{4}$ showed many of the usual clinical features of pulmonary thromboembolism with infarction, except for the unusual features of persistent fever and pleural effusion, along with the finding of pericarditis and the dramatic response to corticosteroids therapy. The onset of the syndrome has been found to be much shorter after 
transvenous pacing, such as pacemaker implantation. A 68-year-old man described by Bajaj et al, ${ }^{5}$ who had a history of presyncopal and syncopal symptoms and who underwent pacemaker implantation, developed signs and symptoms of PCIS within days of his surgery.

That the degree of myocardial damage does not correlate directly with the development of PCIS ${ }^{8}$ implies an immunologic basis for the syndrome. In addition, a seasonal variation has been reported, which suggests a viral cause. Although PCIS has been found to occur after minor procedures, such as transvenous pacing, it has rarely been reported after angioplasty. Velander and colleagues 9 described the first case of PCIS after angioplasty in 1993. They reported a patient who developed Dressler's syndrome after a massive myocardial infarction. Nine years later, that patient underwent angioplasty for progressive angina pectoris, and within 1 week of the procedure, the patient developed PCIS. This case was, however, not straightforward given the patient's previous history of PCIS.

The only other case of PCIS after angioplasty was reported by Escaned and colleagues in $1992 .{ }^{10}$ Their patient suffered coronary perforation during the course of angioplasty. The next day, the patient developed pleuropericarditis. The rapid onset of symptoms in that case, however, mitigates against the diagnosis of PCIS.

\section{Conclusion}

Our case appears to be the first of PCIS occurring after an uncomplicated angioplasty and stenting. The syndrome has been well documented to occur after similar minimally invasive cardiac procedures. Given the frequency with which these procedures are performed, the occurrence of PCIS in this setting is not surprising. One should consider the diagnosis of PCIS when a patient develops fever, myalgias, pleurisy, and pleural effusion after coronary angioplasty and stenting.

\section{References}

1. Khan AH. The postcardiac injury syndromes. Clin Cardiol 1992;15:67-72.

2. Ito T, Engle MA, Goldberg HP. Postpericardiotomy syndrome following surgery for nonrheumatic heart disease. Circulation 1958;17:549-56.

3. Dressler W. A post-myocardial infarction syndrome; preliminary report of a complication resembling idiopathic, recurrent, benign pericarditis. JAMA 1956; 160:1379-83.

4. Sklaroff HJ. The post-pulmonary infarction syndrome. Am Heart J 1979;98:722-6.

5. Bajaj BPS, Evans KE, Thomas P. Postpericardiotomy syndrome following temporary and permanent transvenous pacing. Postgrad Med J 1999;75: $357-8$.

6. Loughlin V, Murphy A, Russell C. The post-pericardiotomy syndrome and penetrating injury of the chest. Injury 1987;18:412-4.

7. Stelzner TJ, King TE Jr, Antony VB, Sahn SA. The pleuropulmonary manifestations of the postcardiac injury syndrome. Chest 1983;84:383-7.

8. Normura Y, Yoshinaga M, Haragushi T, et al. Relationship between degree of injury at operation and the change in antimyosin antibody titer in the postpericardiotomy syndrome. Pediatr Cardiol 1994;15: $116-20$.

9. Velander M, Grip L, Mogensen L. The postcardiac injury syndrome following percutaneous transluminal coronary angioplasty. Clin Cardiol 1993; 16:353-4.

10. Escaned J, Ahmad RA, Shiu MF. Pleural effusion following coronary perforation during balloon angioplasty: an unusual presentation of the postpericardiotomy syndrome. Eur Heart J 1992;13:716-7. 